\title{
Aging in a Model of Self-Organized Criticality
}

\author{
Stefan Boettcher ${ }^{1,2,3}$ and Maya Paczuski ${ }^{4}$ \\ ${ }^{1}$ Center for Theoretical Studies of Physical Systems, Clark Atlanta University, Atlanta, GA 30314 \\ ${ }^{2}$ Department of Physics and Astronomy, The University of Oklahoma, Norman, OK 73019-0225 \\ ${ }^{3}$ Center for Nonlinear Studies, MS-B258, Los Alamos National Laboratory, Los Alamos, NM 87545 \\ ${ }^{4}$ Department of Physics, University of Houston, Houston TX 77204-5506
}

(August 13, 2018)

\begin{abstract}
Temporal autocorrelation functions for avalanches in the Bak-Sneppen model display aging behavior similar to glassy systems. Numerical simulations show that they decay as power laws with two distinct regimes separated by a time scale which is the waiting time, or age, of the avalanche. Thus, time-translational invariance is dynamically broken. The critical exponent of the initial decay is that of the familiar stationary dynamics while a new critical exponent for the late-time behavior appears. This new exponent characterizes a non-stationary regime that has not been previously considered in the context of self-organized criticality. PACS numbers: 64.60.Lx, 05.40.+j, 05.70.Ln
\end{abstract}

Self-organized criticality (SOC) [1] describes a general property of slowly-driven dissipative systems with many degrees of freedom to reach a stationary state where they evolve intermittently in terms of bursts spanning all scales up to the system size. Many natural avalanchelike phenomena have been represented using this idea, including earthquakes extinction events in biological evolution, and landscape formation [2]. Recently, SOC has been observed in controlled laboratory experiments on rice piles [3]. Theoretical models of rice piles [4] are related to a variety of different physical systems by universality [5. The emergence of long-term memory in the self-organized critical state has been demonstrated analytically for a multi-trait evolution model [6], a variant of the Bak-Sneppen model [7].

Here we show that the self-organized critical state in the Bak-Sneppen model exhibits aging behavior that is reminiscent of glassy systems 88. We numerically measure two-time autocorrelation functions for avalanches and observe a power-law decay with two distinct regimes. The early time power law regime is that of the familiar stationary dynamics. The late time regime has a new critical exponent characterizing the history-dependent relaxation behavior of the model. The time scale separating the early and late time regimes is the age of the avalanche. Rescaling the autocorrelations measured in units of the respective age collapses the data onto a scaling function of a single variable. The distinct late time regime signals a breaking of time-translational invariance arising from the system's ability to store information from past events over arbitrarily long times.

"Aging" is an important phenomenon observed experimentally in glassy materials where relaxation behavior depends on the previous history of the system [9]. For instance, consider a spin glass quenched below the glass transition at time $t=0$ in the presence of a small magnetic field. Throughout the sample, domains of various pure states develop that grow with characteristic size
$R(t)$. At time $t=t_{w}$, the magnetic field is turned off and the system's response in the form of its remanent magnetization is measured. Initially, the response function is only sensitive to the pure, quasi-equilibrium states in their distinct domains. But after an additional time scale related to the waiting time, $t_{w}$, the response spans entire domains and slows down when it experiences the non-equilibrium state the sample possesses as a whole. A simple scaling form holds for some glassy systems [10]. The autocorrelation function for the remanent magnetization is given by

$$
C\left(t+t_{w}, t_{w}\right)=t^{-\mu} f\left(t / t_{w}\right)
$$

where $f(x \rightarrow 0) \rightarrow$ constant and $f(x>>1) \sim x^{-r}$ with $r>0$. Similar aging behavior also appears in a variety of other problems such as coarsening dynamics, directed polymers in random media, etc. 11.

Generally, this type of correlation function can be measured for avalanches in the self-organized critical state (which will be specified below for the Bak-Sneppen model). Here, we define the waiting time, $t_{w}$, to be the time since the beginning of the avalanche. In order to eliminate trivial effects of norm conservation [12] referring to the fact that avalanches die, we only consider the dynamics of infinite avalanches, or those that survive longer than any time scale we consider. We focus on a simple quantity, $P_{\text {first }}(t)$, measuring the first returns of the activity to a given site. A power law distribution for $P_{\text {first }}(t)$ has been measured numerically for a variety of different SOC models [13] by recording first returns to every site visited during the avalanche. Here we measure the probability $P_{\text {first }}\left(t_{w} ; t\right)$ for the infinite avalanche to return after $t$ time steps to a site that was visited most recently at time $t_{w}$ from the beginning of the avalanche. Thus, to determine the first-return probability, we take the age of the avalanche, $t_{w}$, into account. While in the stationary state of SOC models the first return distribution is generally $P_{\text {first }}(t) \sim t^{-\tau_{\text {first }}},(t \rightarrow \infty)$, we find for 
the Bak-Sneppen model

$$
\begin{aligned}
P_{\text {first }}\left(t_{w} ; t\right) \sim t^{-\tau_{\text {first }}} f\left(\frac{t}{t_{w}}\right) & \\
& f(x) \sim \begin{cases}\text { constant } & (x \ll 1), \\
x^{-r} & (x \gg 1) .\end{cases}
\end{aligned}
$$

The exponent $\tau_{\text {first }}$ can be related to other critical exponents via scaling relations for SOC [13]. The origin of the coefficient $r$ is non-trivial, signaling the dynamical breaking of time-translational invariance in the infinite avalanche. (For instance, the first-return probability to any site $n$ for a random walker that started at the origin at time $t^{\prime}=0$ and passed site $n$ at time $t_{w}$ is clearly independent of $t_{w}$ unless time translational invariance is explicitly broken.) In glassy systems, the coefficient $r$ usually refers to off-equilibrium properties of the system [8]. The question then arises whether the coefficient $r$ in Eq. (2) can be related to the known universal coefficients of the stationary SOC process, or whether it describes new physics in the avalanche dynamics of the Bak-Sneppen model, and possibly other models of SOC.

The Bak-Sneppen model [7] has been studied intensely and with great numerical accuracy in recent years. We refer to Ref. 13 for a review of its many features and simply utilize those facts here. The model consists of random numbers $\lambda_{i}$ between 0 and 1, each occupying a site $i$ on a lattice in, say, $d=1$. At each update step, the smallest random number $\lambda_{\min }(t)$ is located. That site as well as its two nearest neighbors each get new random numbers drawn independently from a flat distribution between zero and one. The system evolves to a SOC state where almost all numbers have values above $\lambda_{\mathrm{c}}$, with $\lambda_{\mathrm{c}}$ avalanches formed by the remaining numbers below. In our simulations, we have used the equivalent branching process [14 with $\lambda_{\mathrm{c}}=0.66702$ to eliminate any finite-size effects. Initially, at time $t_{a}=0$, the smallest threshold value is set equal to $\lambda_{c}$ to start a $\lambda_{\mathrm{c}}$ avalanche. In every update $t_{a} \rightarrow t_{a}+1$, only the signal $\lambda_{\min }\left(t_{a}\right)$ and its two nearest neighbors receive new threshold values. At any time, we store only those threshold values $\lambda_{i}<\lambda_{\mathrm{c}}$ that are part of the avalanche because only those numbers can contribute to the signal, i. e. can ever become smallest number. In addition, we keep a dynamic list of every site that has ever held the signal at some time to determine the first-return probabilities. It has been shown that the Bak-Sneppen model in $d=1,2$ is a fractal renewal process 13 , i. e. activity is recurrent for each site unless the avalanche dies. Avalanches end when there are no $\lambda_{i}<\lambda_{\mathrm{c}}$, and a new (independent) avalanche is initiated with $t_{a}=0$. If the avalanche survived longer than $t_{c o}=2^{27}$ then we store the data for the first returns; otherwise the data is discarded. This way, we probe properties of infinite avalanches up to the cut-off time scale $t_{c o}$.

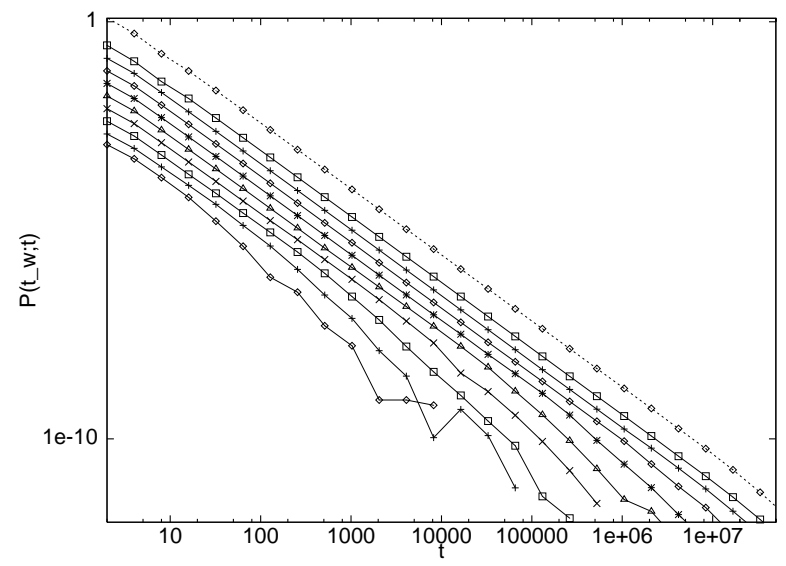

FIG. 1. Log-log plot of the first-return probabilities $P_{\text {first }}\left(t_{w} ; t\right)$ as a function of $t$ for various $t_{w}$ in the one dimensional Bak-Sneppen model. The graphs are consecutively labeled from bottom to top by $i=1,2, \ldots, 9$ where each graph contains data for all $8^{i-1} \leq t_{w} \leq 8^{i}-1$. To avoid overlaps, each distribution is offset by a factor of $2^{i}$. Initially, each graph falls like a power law with the familiar coefficient $\tau_{\text {first }}=1.58$. But for values of $t \gg t_{w}$, each graph crosses over into a new power law regime with a larger coefficient. For reference, we have also plotted the first return distribution for the combined data as a dashed line on the right. Note the smooth power law behavior over six decades until cut-off effects set in at about $10^{7} \approx 0.1 t_{\mathrm{co}}$.

At each update $t_{a}$ we determine the previous time $t_{w}$ when the signal was on the same site most recently (if ever). Then its first-return time is give by $t=t_{a}-t_{w}$, and we bin histograms labeled by $i=\left\lceil\frac{1}{3} \log _{2} t_{w}\right\rceil$ and $j=\left\lceil\log _{2} t\right\rceil$. The data is binned logarithmically so that in each bin a comparable number of data points is averaged over: for each increment of $j$, the width of the bins for $t$ increases by a factor of 2 , while for each increment of $i$ the $t_{w}$ bins increase by a factor of 8 . $P_{\text {first }}\left(t_{w} ; t\right)$ for various values of $t_{w}$, and $P_{\text {first }}(t)$ obtained from the combined data, are plotted in Fig. 11. Each graph refers to a different range for $t_{w}$, increasing by a factor of 8 each time from left to right. Each graph possesses two distinct power law regimes, separated by a crossover. To determine the form of the scaling function $f(x)$ for these graphs according to Eqs. (2), we note that the crossover appears to scale linearly with $t_{w}$. Thus, we plot

$$
f(x) \sim t^{\tau_{\text {first }}} P_{\text {first }}\left(t_{w} ; t\right) \quad \text { with } \quad x=\frac{t}{t_{w}}
$$

using $\tau_{\text {first }}=1.58$ [13], see Fig. 2. The data collapses reasonably well onto a single curve, $f(x)$, which is constant for small argument, and appears to fall like a power law with coefficient $r=0.45 \pm 0.10$ over two decades. The 
numerical value for $r$ is difficult to extract with greater accuracy because it refers to properties deep within the tail of the distribution, but it is sufficiently accurate to prove the existence of a distinctly new regime at time scales beyond $t_{w}$, indicating aging behavior. The data excludes the possibility that the effect is due to some hidden cut-off (an exponential would have to be extremely weak to fall for less than a decade over two decades in its argument), or due to mere statistical noise. Similar results were obtained for the two dimensional Bak-Sneppen model where we find $r=0.25 \pm 0.10$. Although a smaller value of $\tau_{\text {first }}=1.28$ in the two dimensional Bak-Sneppen model leads to many more events in the tail of the autocorrelation functions, our numerical results in that case are less conclusive due to apparent significant corrections to scaling in the distribution for the first returns [17.

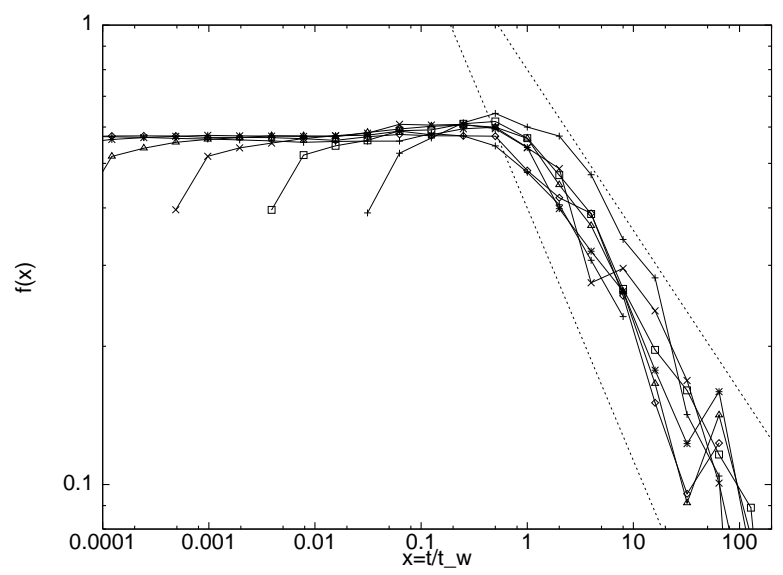

FIG. 2. Data collapse according to Eq. (3) for the data in Fig. 1 (using the same symbols), discarding data for $i=1$ and $i=9$ to eliminate short-time and cut-off effects, respectively. The scaling function $f(x)$ is constant for small argument and appears to follow a power law for $x \gg 1$ for about two decades before the data gets too noisy. The two dashed lines that bracket the power law tail correspond to $x^{-0.35}$ and $x^{-0.55}$. We estimate $r=0.45 \pm 0.10$.

As written, the scaling theory of Ref. [13] does not describe the origin of the coefficient $r$ since it considers only stationary properties of avalanches where the breaking of time translational invariance does not appear. We have considered domain growth approaches to explain the origin of aging, without success. For instance, the activity in an evolving avalanche spreads over a domain that is growing with a characteristic length scale $R(t) \sim t^{1 / D}$ [13. Thus, between $t_{w}$ and $t_{w}+t$, the domain covered by the avalanche has grown and might have decreased the probability to return to a particular site by a fraction $\left(1+t / t_{w}\right)^{-d / D}$, implying $r=d / D$. For $d=1, D \approx 2.43$ [13], which agrees well with the result for $r$, but for $d=2$, $D \approx 2.92$, which is inconsistent with the above result for $r$. However only few sites inside an avalanche domain are actually part of the avalanche at any one time, their number merely growing like $t^{d_{s}}$ with $d_{s} \approx 0.11$ and 0.25 in $d=1$ and 2 [13. Assuming that $r=d_{s}$ is inconsistent with the measured value of $r$ for $d=1$. While similar geometrical arguments may quantitatively explain observed aging behavior in systems whose dynamics is dominated by the coarsening of domains [15, it appears that aging in the Bak-Sneppen model and possibly other SOC models is more intricately linked to the long term memory of the process which can distinguish sites that were last visited early in the avalanche from those visited much later.

We have also investigated the possibility of aging in other SOC models such as the Abelian sandpile due to Bak, Tang, and Wiesenfeld (BTW) [1] and the Manna two-state sandpile model [16], which will be reported in detail elsewhere 17. In short, numerical measurements of the first return probabilities for the Manna model show no sign of any age dependence at all. The BTW model [1] does show nontrivial aging behavior which is quite different from that of the Bak-Sneppen model, i.e. neither its scaling variable nor its scaling function is as simple as Eq. (2). The appearance of aging in the BTW model demonstrates at least a weak memory not included in the discussion of Ref. [19]. Despite the gross similarities between the BTW and the Manna model, our results suggest that the avalanche dynamics in both models is completely different; in fact they have been found to belong to different universality classes [18]. We do not know yet to what extent aging is a general property of self-organized critical systems.

It is well known from the study of amorphous materials that systems with hierarchically ordered dynamics, where slow degrees of freedom can only advance after faster ones have moved collectively, typically lead to slow relaxation behavior [20], similar to the power-law distributed avalanches in SOC. For instance, frustration has been identified as a cause for slow relaxation in spin systems, requiring cooperative behavior between ever more distant spins to further decrease their collective energy. Phenomenological models of relaxation in glassy systems 21] attribute slow relaxation and aging to the complicated phase-space structure, with a highly degenerate equilibrium state, and with a hierarchy of ever-higher barriers slowing the approach to any state of lower energy, that originates from the inherent randomness in the microscopic interactions. Previously, explicitly hierarchical models of diffusion on ultrametric trees have been invoked to describe experimental findings for such glassy systems [22], including their aging behavior [23].

Remarkably, the Bak-Sneppen mechanism shares many of these "glassy" features. The far-from- 
equilibrium dynamics is characterized by a large pool of virtually inert ("frozen") threshold values, equally distributed above $\lambda_{c}$, assuming one among an infinite degeneracy of quasi-stable configurations that the process is attracted to. The update process perturbs the system, inducing avalanches which are relaxations events towards a new such configuration. In avalanche dynamics, active ("fast") threshold values achieve improved stability only through the cooperative behavior of neighboring sites. A critical avalanche can only end through a longrange cooperation of many sites. These avalanches, as in all extremal models of SOC, consist of a hierarchy of sub-avalanches which are temporally and spatially contained within their parent avalanches [13]. In Ref. [6] we showed how this hierarchical structure in the analytically tractable multi-trait model leads to the build-up of memory during an avalanche. There, we also explored the ultrametric properties of the self-organized critical state, and found that the ultrametric separation between consecutive minima evolves to be power-law distributed. Thus, while imposed explicitly to describe aging in glassy systems in Refs. [23], hierarchical or ultrametric structures emerge dynamically in some models of SOC and apparently lead to similar aging behavior.

Our numerical results demonstrate that aging occurs not only in glasses and other out of equilibrium systems slowly approaching their asymptotic equilibrium state, but also occurs in some models of self-organized criticality. Aside from glasses or SOC, there exists a much wider range of important problems exhibiting certain aspects of glassy behavior, for example the directed polymer [11, protein folding, learning, or optimization algorithms [24]. The dynamics of these problems is poorly understood and often conceptualized in terms of a walk through a "rugged landscape" 25]. More than adding another problem to that list, we believe that the avalanche dynamics of some SOC models is far more accessible, with many subtle features considering their simplicity, that they might provide a basic framework to study the emergence of slow relaxation and aging. This is certainly possible if the mechanism responsible for aging is avalanche dynamics in systems approaching a critical state.

We acknowledge helpful correspondences with Mike Creutz. SB acknowledges partial support under DOE grant DE-FE02-95ER40923.

[1] P. Bak, C. Tang, and K. Wiesenfeld, Phys. Rev. Lett. 59, 381 (1987); Phys. Rev. A. 38, 364 (1988).

[2] For a review see P. Bak, How Nature Works: The Science of Self-Organized Criticality, (Copernicus, New York, 1996).

[3] V. Frette, K. Christensen, A. Malthe-Sørenssen, J. Feder, T. Jøssang, and P. Meakin, Nature 379, 49 (1996).

[4] K. Christensen, A. Corral, V. Frette, J. Feder, and T. Jøssang, Phys. Rev. Lett. 77, 107 (1996).

[5] M. Paczuski and S. Boettcher, Phys. Rev. Lett. 77, 111 (1996).

[6] S. Boettcher and M. Paczuski, Phys. Rev. E 54, 1082 (1996), and Phys. Rev. Lett. 76, 348 (1996).

[7] P. Bak and K. Sneppen, Phys. Rev. Lett. 71, 4083 (1993).

[8] E. Vincent, J. Hammann, M. Ocio, J.-P. Bouchaud, and L. F. Cugliandolo, in Proceeding of the Sitges Conference on Glassy Systems, ed. E. Ruby (Springer, Berlin, 1996), and preprint cond-mat/9607224; G. Parisi, Nuovo Cimento D 16, 939 (1994); J. Kurchan and L. Laloux, J. Phys. A 29, 1929 (1996).

[9] E. Vincent, J. Hammann, and M. Ocio, in Recent Progress in Random Magnets, ed. D. H. Ryan, (World Scientific, Singapore, 1992).

[10] H. Rieger, J. Phys. A 26, L615, (1993); Physica A 224, 267 (1996); L. F. Cugliandolo and J. Kurchan, Phys. Rev. Lett. 71, 1 (1993).

[11] H. Yoshino, J. Phys. A 29, 1421 (1996); A. Barrat, Phys. Rev. E 55, 5651 (1997); S. Franz and J. Hertz, Phys. Rev. Lett. 74, 2114 (1995); J. Kurchan, L. Peliti, and M. Sellitto, preprint cond-mat/970414d; L. Frachebourg, P. L. Krapivsky, and S. Redner, Phys. Rev. E 55, 6684 (1997).

[12] D. A. Stariolo, Phys. Rev. E 55, 4806 (1997).

[13] M. Paczuski, S. Maslov, and P. Bak, Phys. Rev. E 53, 414 (1996).

[14] M. Paczuski, S. Maslov, and P. Bak, Europhys. Lett. 27, 97.

[15] A. Barrat, R. Burioni, and M. Mezard, J. Phys. A 29, 1311 (1996).

[16] S. S. Manna, J. Phys. A: Math. Gen. 24, L363 (1991).

[17] S. Boettcher and M. Paczuski, (in preparation).

[18] A. Ben-Hur and O. Biham, Phys. Ref. E 53, R1317 (1996).

[19] M. Marsili, G. Caldarelli, and M. Vendruscolo, Phys. Rev. E 53, R13 (1996).

[20] R. G. Palmer, D. L. Stein, E. Abrahams, and P. W. Anderson, Phys. Rev. Lett. 53, 958 (1984).

[21] J. P. Bouchaud, J. Phys. I France 21705 (1992).

[22] A. T. Ogielski and D. L. Stein, Phys. Rev. Lett. 55, 1634 (1985); M. Schreckenberg, Z. Phys. B 60, 483 (1985).

[23] P. Sibani and K. H. Hoffmann, Phys. Rev. Lett. 63, 2853 (1989); K. H. Hoffmann and P. Sibani, Phys. Rev. A 38, 4261 (1988); H. Yoshino, J. Phys. A 30, 1143 (1997); H. Rieger, preprint cond-mat/9703235.

[24] M. Mezard, G. Parisi, and M. A. Virasoro, Spin Glass Theory and Beyond (World Scientific, Singapore, 1987); see also Landscape Paradigms in Physics and Biology, Proceedings of the 16th Annual International Conference at $C N L S$, (to appear in Physica D).

[25] For an attempt to relate "rugged landscapes" and SOC, see D. L. Stein and C. M. Newman, Phys. Rev. E 51, 5228 (1995). 